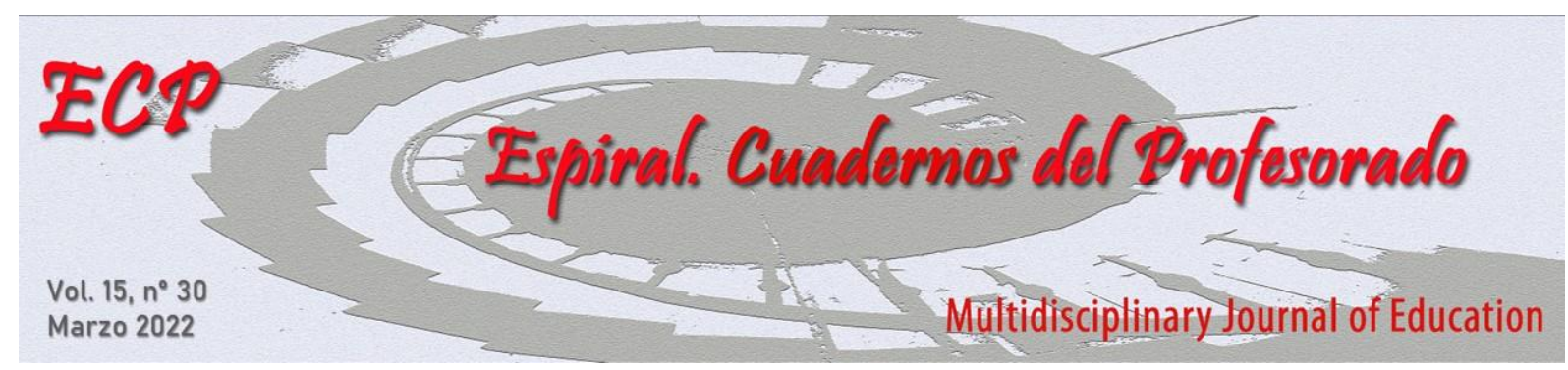

\title{
Perfil y percepciones de los estudiantes del Máster universitario en formación del profesorado de educación secundaria en España
}

\section{Profile and perceptions of the students of the Master in secondary education teacher training in Spain}

\author{
Roberto Sánchez-Cabrero y Francisco Javier Pericacho-Gómez
}

Universidad Autónoma de Madrid, España

\begin{abstract}
Resumen
Los debates en torno a la mejora de la calidad educativa tienen un lugar privilegiado en las agendas políticoeducativas nacionales e internacionales. La Unión Europea ha expresado su compromiso con la mejora de la profesión docente, destacando la formación inicial como un pilar fundamental. El objetivo de este estudio es conocer el perfil y la percepción del alumnado del Master de Formación del Profesorado en España sobre una serie de variables relacionadas con dicha formación y el perfil profesional. Para ello, se realiza un estudio descriptivo correlacional del perfil social y formativo de 381 alumnos del Máster de Formación del Profesorado de una universidad española. Se elaboró un cuestionario informatizado ad hoc en el que se miden doce variables y se analizan las correlaciones mediante Chi-Cuadrado y $d$ de Somers sobre tablas de contingencias. Los resultados muestran un perfil claramente vocacional y una buena imagen del docente y su formación.
\end{abstract}

Palabras clave: Formación inicial profesorado; máster en Educación Secundaria; formación de docentes; percepción.

\begin{abstract}
Debates regarding the improvement of educational quality have a privileged place in the national and international political-educational agendas. The European Union has expressed its commitment to improving the teaching profession, highlighting initial training as a fundamental pillar. The objective of this study is to know the profile and perception of the students of the Master of Teacher Training in Spain on a series of variables related to said training and the professional profile. For this, a correlational descriptive study of the social and training profile of 381 students of the Master's Degree in Teacher Training is carried out. An ad hoc computerized questionnaire was developed in which twelve variables are measured and correlations are analyzed using Chi-Square and Somers's $d$ on contingency tables. The results show a clearly vocational profile and a good image of the teacher and their training.
\end{abstract}

Keywords: Initial teachers training; master of secondary teachers; teacher education; perception.

Fecha de recepción: 15/04/2021

Fecha de aceptación: 12/07/2021

Correspondencia: Roberto Sánchez-Cabrero, Universidad Autónoma de Madrid, España Email: roberto.sanchez@uam.es 


\section{Introducción}

La formación inicial del profesorado de Educación Secundaria constituye un motivo de gran preocupación en todos los países de Europa (Hendriks et al., 2010, Rodríguez et al., 2019) y, en general, en todo el ámbito de los estados pertenecientes a la OECD (Organisation for Economic Co-operation and Development). En un conocido estudio desarrollado por la OECD (2004) se evidenció que en la mayoría de los países desarrollados la cualificación de los docentes tenía un significativo margen de mejora. En este sentido, la Unión Europea y sus Estados miembros han expresado su firme compromiso con la mejora de la profesión docente (Manso et al., 2019), destacando, entre todos los elementos que la constituyen, la formación inicial como un pilar fundamental. El firme convencimiento de que en los docentes se encuentra una parte esencial de la calidad educativa ha generado la reflexión y el replanteamiento de su formación inicial (Ball y Forzani, 2009; Cochran y Zeichner, 2009).

En la literatura científica existe un amplio consenso sobre la importancia de los programas de formación inicial del profesorado y su relación con la calidad de la enseñanza y el aprendizaje en los alumnos (Darling- Hammond, 2017; Fernández et al., 2016; Marcelo, 2010). Igualmente, informes de gran relevancia internacional como el Informe McKinsey (Barber y Mourshed, 2008) ponen de manifiesto que un factor relevante asociado al éxito de un modelo educativo es la formación de sus docentes (bajo el cual puede determinarse en gran medida el éxito o el fracaso del modelo educativo), y que los países con los sistemas educativos más efectivos del mundo son también los que más énfasis ponen en cuidar la formación inicial y el desarrollo profesional de sus docentes.

Singularmente, en el contexto español la trayectoria histórica de la formación inicial docente denota un creciente interés por su mejora. La configuración actual partió de la incorporación de España al Espacio Europeo de Educación Superior (EEES) que dio inició a partir de la Declaración de Bolonia en 1999 firmada por 29 ministros europeos de educación. El EEES configuró las líneas maestras de lo que debía ser un marco educativo común europeo para la educación superior y por el que España actualizó y transformó sus titulaciones universitarias (Álvarez et al., 2009). Como se pone de manifiesto, la situación en España actualmente es significativamente mejor que la existente con la formación estandarizada anterior, el denominado como 'CAP' (Certificad de Aptitud Pedagógica) (Perales et al., 2014; Pontes et al., 2015), que en algunos casos incluso tenía una duración inferior a un trimestre escolar y que ha sido la única formación psicopedagógica que recibieron muchos de los docentes de secundaria que a día de hoy siguen ejerciendo, puesto que fue el formato oficial hasta el curso 2009-2010.

La evidencia científica pone de manifiesto que los estudiantes inician los programas de formación inicial con ideas preconcebidas y bien arraigadas acerca de lo que constituye el proceso de enseñanza y aprendizaje, fruto de sus experiencias propias y de su trayectoria cultural (Beyer y Zeichner, 2018; Romero y Trigo, 2018; Serrano y Pontes, 2015a; Zapico et al., 2017). Estas experiencias funcionan como filtros que pueden condicionar su modo de comprensión del conocimiento que reciben en los programas de formación inicial, y también sus creencias acerca de la práctica docente (Biesta et al., 2015). En este contexto, cabe preguntarse si un alumno del actual Máster de Formación de Profesorado de Secundaria, que inicialmente parte con una carencia casi total de conocimientos didácticos previos (Serrano y Pontes, 2015b), recibe una formación suficiente como para afrontar con altas garantías de éxito su labor profesional (Manso y Martín, 2014). Esta preocupación, en el contexto Europeo, alberga una significativa producción científica que evidencia tres necesidades fundamentales: en primer lugar, mejorar los procesos de selección de los candidatos a los programas de formación inicial en cada país; en segundo lugar, equilibrar el peso formativo entre la formación pedagógica general y la propia de cada disciplina; por último, consolidar una fase de prácticas de calidad donde los estudiantes realmente puedan mejorar sus competencias (Hendriks et al., 2010; Manso et al., 2019; Muñoz et al., 2019; Romero y Trigo, 2018).

Esta preocupación, no solo se refleja en la comunidad científica, también es evidenciada por el propio alumnado, como se pone de manifiesto en el estudio de Benarroch et al. (2013) en el que, por ejemplo, se destaca la necesidad de recibir conocimientos instrumentales. También se puede observar en Manso y Martín (2014), que constatan cómo lo más valorado por los alumnos son los aprendizajes relacionados con las didácticas específicas. En este sentido, estudios recientes como el Sola et al. (2020) destacan que "los estudiantes otorgan gran valor a aquellas cuestiones vinculadas a la competencia

Espiral. Cuadernos del Profesorado | ISSN 1988-7701 | 2022, 15(30), 71-83 
aprender a aprender, la importancia de la adecuada convivencia y relaciones sociales, así como el fomento de un espíritu crítico y reflexivo" (2020, p. 81).

Por otra parte, el conocimiento científico nacional e internacional acumulado sugiere dos puntos fundamentales a tener presente en las investigaciones que se realicen sobre la temática. En primer lugar, entender la formación inicial docente como un momento complejo, especialmente sensible y con gran repercusión en la construcción del futuro docente (Muñoz et al., 2019; OCDE, 2019; Sola et al., 2020). En segundo lugar, la necesidad de seguir aumentando, complejizando y actualizando el conocimiento sobre el alumnado que participa en dichos programas de formación inicial docente (Benarroch et al., 2013; Biesta et al., 2015; Cochran y Zeichner, 2009; Darling- Hammond, 2017).

En este contexto y ante la evidencia expuesta, la principal aportación de nuestra investigación consiste en seguir profundizando, actualizando y aumentando el conocimiento sobre el alumnado del Máster de Formación de Profesorado en España, concretamente sobre su perfil social y formativo, interés en la docencia, percepción del Máster y la imagen social que percibe de la profesión docente en la sociedad, entre otras cuestiones. Sin duda constituye una fuente de información indispensable y muy útil a la hora de reflexionar, interpretar, perfilar y orientar con mayor criterio posibles medidas de mejora e innovación en la formación inicial de los docentes.

En consecuencia, todas estas cuestiones planteadas perfilan el sentido, implicaciones prácticas, contribución al conocimiento disponible y finalidad de esta investigación, que se plantea tres objetivos principales. En primer lugar, (1) definir el perfil social y formativo de los alumnos del Máster de Formación del Profesorado en España. En segundo lugar, (2) reflejar la visión que los alumnos tienen de su propia formación. En tercer lugar, (3) evaluar la imagen que refleja la profesión docente a través de las propias experiencias del alumno, su situación actual y sus expectativas ante el futuro.

\section{Método}

\section{Participantes}

La muestra del estudio está formada por 381 participantes (235 mujeres y 146 varones), alumnos del Master de Formación del Profesorado de Secundaria oficial, con una edad media de 34,49 años (34,17 para mujeres y 35,01 para hombres) y una desviación típica de 7,01 (6,91 para mujeres y 7,17 para hombres). A continuación, en la Figura 1, puede observarse de manera visual la distribución de la muestra teniendo en cuenta la edad y el sexo.

\section{Figura 1.}

Pirámide poblacional de la muestra obtenida teniendo en cuenta la edad y el sexo de los participantes.

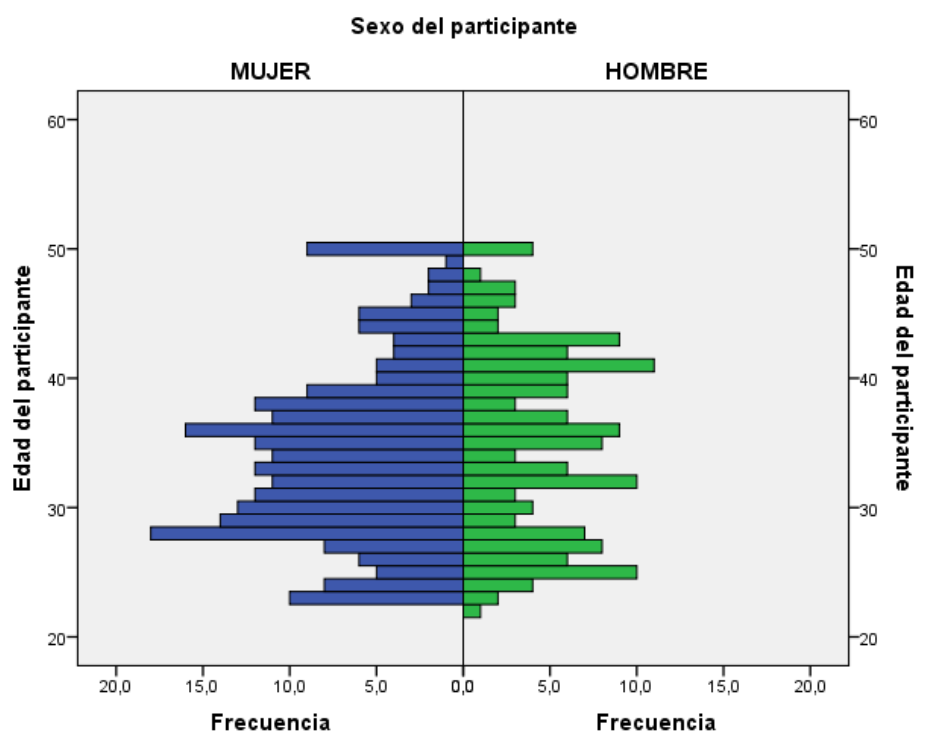

Espiral. Cuadernos del Profesorado | ISSN 1988-7701 | 2022, 15(30), 71-83 
La muestra ha sido obtenida de las ocho áreas de matrícula diferentes en el Master de Formación del Profesorado de Secundaria disponibles (Lengua y Literatura, Ingles, Matemáticas, Biología y Geología, Física y Química, Tecnología, Geografía e Historia y Economía), siendo la modalidad de Economía (N: 92) la más frecuente y la de Inglés (N: 20) la menos frecuente como puede observarse en la Figura 2 a continuación.

Figura 2.

Distribución de la muestra según área o especialidad seleccionada.

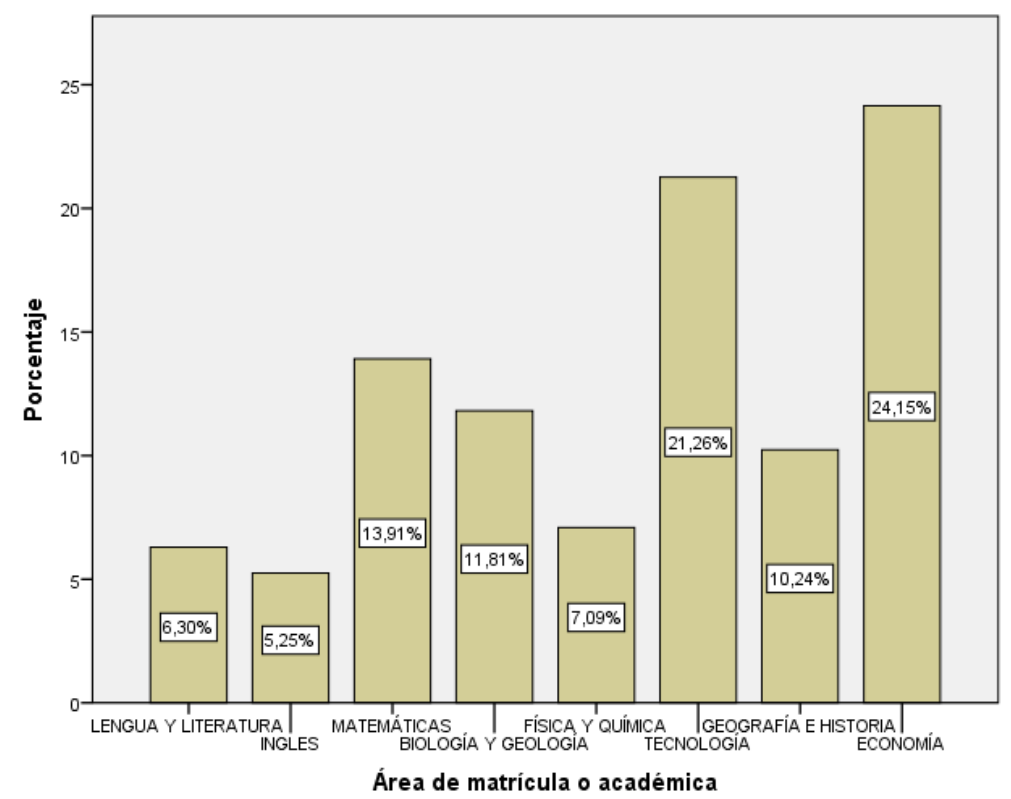

El muestreo ha sido realizado por conglomerado, a través del Master de Formación del Profesorado de Secundaria online de la Universidad Alfonso X el Sabio. No se ha aplicado ningún criterio de selección extra al de estar cursando el Master de Formación del Profesorado de Secundaria, por lo que la muestra es heterogénea en perfiles profesionales y proviene de distintas comunidades de todo el Estado Español.

\section{Instrumentos}

Se ha diseñado un cuestionario informatizado ad hoc alojado en el servidor privado de Encuestafacil.com para que los participantes pudieran acceder a él a distancia desde cualquier tipo de dispositivo electrónico con acceso a internet. El cuestionario ha sido validado por el Comité Científico y Ético de la Universidad Alfonso X el Sabio y ha superado un estricto proceso de validación mediante expertos externos.

El cuestionario fue aplicado a mitad del curso académico 2018-2019 del Máster, justo al inicio del segundo cuatrimestre, para garantizar que todos los participantes estuvieran en un punto intermedio del proceso de convertirse en docentes de secundaria. Constaba de 2 páginas y 12 cuestiones, siendo la primera página para la aceptación del consentimiento informado del participante y la última para evaluar las variables consideradas en el estudio. Las 12 cuestiones/variables evaluadas son: Edad, Sexo, Área académica de formación previa, Interés vocacional en la docencia, Interés a corto plazo en el Master de Formación del Profesorado de Secundaria (hace referencia a la existencia o no de un interés claro del participante en la obtención del título del Master de Formación del Profesorado de Secundaria. Por ejemplo, la realización de unas oposiciones para la obtención de empleo público, o la existencia de una oferta de empleo concreta), Experiencia docente, Recuerdo positivo o negativo de la formación de los docentes de secundaria cuando el participante fue estudiante de secundaria, Valoración de la suficiencia del actual Master de Formación del Profesorado de Secundaria para hacer frente a todas las necesidades formativas profesionales, Valoración de la necesidad de ampliación de la formación inicial de los docentes de secundaria, Disponibilidad del participante para ampliar su formación obligatoria para ejercer de 
docente de secundaria, Imagen personal del docente de secundaria e Imagen del docente de secundaria en la sociedad.

\section{Diseño y procedimientos}

Se ha realizado un estudio descriptivo y correlacional de corte transversal de la realidad y reflexiones del alumnado del Máster de Formación de Profesorado, relacionadas con su futuro ámbito laboral, valorando la influencia e interacción de distintas variables nominales y ordinales y cuantitativas. Una vez obtenidos los datos de los participantes de forma anónima, se ha procedido a los análisis estadísticos mediante la herramienta estadística informatizada de IBM SPSS (Statistical Package for the Social Sciences).

Se ha utilizado la media aritmética y la desviación típica como estadísticos descriptivos en la variable cuantitativa 'Edad', y la distribución de frecuencias en el resto de variables. Para los análisis inferenciales, se ha procedido a convertir la variable cuantitativa discreta 'Edad' a variable ordinal con cuatro posibles condiciones, para favorecer la comparación con el resto de contrastes entre las distintas variables, puesto que el resto de variables son nominales u ordinales. Se ha realizado análisis de las contingencias entre las distintas variables a través de Chi-cuadrado, que refleja la significación de la no independencia de las variables medidas, y de la $d$ de Somers, que refleja la orientación de dicha relación, el tamaño del efecto y su significación. Se ha elegido estos estadísticos por ser los más adecuados para utilizar con análisis de contingencias - en el caso de Chi-Cuadrado -, y por ser el estadístico que mejor compara dos variables ordinales, reflejando, por otro lado, la orientación de las diferencias - en el caso de la $d$ de Somers - (Sánchez-Cabrero et al., 2019). Por último, se ha tomado decisiones en relación a la significación con grados de confianza al $99 \%(\alpha: 0,01)$ y $95 \%(\alpha: 0,05)$ en los resultados obtenidos.

\section{Resultados}

En relación con los resultados meramente descriptivos del estudio, la Tabla 1 muestra la distribución de frecuencias de la muestra, según las distintas variables nominales y ordinales consideradas, y la media y desviación típica de la edad de los participantes según las diferentes condiciones.

Tabla 1.

Descripción del contenido de la tabla.

\begin{tabular}{lccc}
\hline Edad del participante & Frecuencia & Porcentaje & Edad: $\boldsymbol{X}(\boldsymbol{d} \boldsymbol{t})$ \\
\hline Menor de 30 & 110 & 28,9 & $26,35(2,01)$ \\
De 30 a 39 & 177 & 46,5 & $34,47(2,78)$ \\
De 40 a 49 & 81 & 21,3 & $43,10(2,39)$ \\
50 o más & 13 & 3,4 & $50,00(0,00)$ \\
\hline Sexo del participante & Frecuencia & Porcentaje & Edad: $\boldsymbol{X}(\boldsymbol{d} \boldsymbol{t})$ \\
\hline Mujer & 235 & 61,7 & $34,17(6,90)$ \\
Hombre & 146 & 38,3 & $35,01(7,17)$ \\
\hline Área académica de formación previa & Frecuencia & Porcentaje & Edad: $\boldsymbol{X}(\boldsymbol{d} \boldsymbol{t})$ \\
\hline Lengua y Literatura & 24 & 6,3 & $34,54(6,43)$ \\
Ingles & 20 & 5,2 & $30,20(6,44)$ \\
Matemáticas & 53 & 13,9 & $34,36(7,01)$ \\
Biología y Geología & 45 & 11,8 & $33,56(6,05)$ \\
Física y Química & 27 & 7,1 & $32,04(5,80)$ \\
Tecnología & 81 & 21,3 & $37,52(6,21)$ \\
Geografía e Historia & 39 & 10,2 & $32,72(8,21)$ \\
Economía & 92 & 24,1 & $34,75(7,27)$ \\
\hline Docencia como vocación & Frecuencia & Porcentaje & Edad: $\boldsymbol{X}(\boldsymbol{d} \boldsymbol{t})$ \\
\hline No & 70 & 18,4 & $34,11(5,83)$ \\
Sí & 311 & 81,6 & $34,57(7,26)$ \\
\hline Interés a corto plazo en el Master & Frecuencia & Porcentaje & Edad: $\boldsymbol{X}(\boldsymbol{d} \boldsymbol{t})$ \\
\hline No definido (nuevas opciones, vocación) & 213 & 55,9 & $35,16(7,11)$ \\
\hline
\end{tabular}

Espiral. Cuadernos del Profesorado | ISSN 1988-7701 | 2022, 15(30), 71-83 
Perfil y percepciones de los estudiantes del Máster universitario en formación del profesorado de educación secundaria en España

\begin{tabular}{|c|c|c|c|}
\hline Empleo a corto plazo (oposiciones, empleo priv.) & 168 & 44,1 & $33,63(6,81)$ \\
\hline Experiencia docente & Frecuencia & Porcentaje & Edad: $X(d t)$ \\
\hline Ninguna & 132 & 34,6 & $33,49(6,01)$ \\
\hline Menos de 1 año & 76 & 19,9 & $32,92(6,29)$ \\
\hline Más de un año y menos de 5 & 117 & 30,7 & $34,93(7,56)$ \\
\hline Más de 5 & 56 & 14,7 & $38,25(7,66)$ \\
\hline Recuerdo de la formación de los docentes cuando fue alumno & Frecuencia & Porcentaje & Edad: $X(d t)$ \\
\hline No & 129 & 33,9 & $33,54(7,37)$ \\
\hline Sí & 252 & 66,1 & $34,97(6,79)$ \\
\hline $\begin{array}{l}\text { Valoración del actual Master para hacer frente a todas las } \\
\text { necesidades }\end{array}$ & Frecuencia & Porcentaje & Edad: $X(d t)$ \\
\hline Insuficiente & 9 & 2,4 & $35,00(7,53)$ \\
\hline Aceptable & 246 & 64,6 & $34.11(6,69)$ \\
\hline Excelente & 126 & 33,1 & $35,19(7,57)$ \\
\hline $\begin{array}{l}\text { Valoración de la necesidad de ampliación de la formación } \\
\text { inicial }\end{array}$ & Frecuencia & Porcentaje & Edad: $X(d t)$ \\
\hline No & 225 & 59,1 & $34,40(6,85)$ \\
\hline Sí & 156 & 40,9 & $34,62(7,25)$ \\
\hline Disponibilidad del participante para ampliar su formación & Frecuencia & Porcentaje & Edad: $X(d t)$ \\
\hline No & 224 & 58,8 & $35,12(6,99)$ \\
\hline Sí & 157 & 41,2 & $33,59(6,97)$ \\
\hline Imagen personal del docente de secundaria & Frecuencia & Porcentaje & Edad: $X(d t)$ \\
\hline Pésima & 0 & 0 & $0(0)$ \\
\hline Mala & 12 & 3,1 & $33,17(7,06)$ \\
\hline Indiferente & 24 & 6,3 & $34,50(6,70)$ \\
\hline Buena & 287 & 75,3 & $34,71(6,75)$ \\
\hline Excelente & 58 & 15,2 & $33,64(8,35)$ \\
\hline Imagen del docente de secundaria en la sociedad & Frecuencia & Porcentaje & Edad: $X(d t)$ \\
\hline Pésima & 8 & 2,1 & $31,00(6,40)$ \\
\hline Mala & 104 & 27,3 & $32,78(6,78)$ \\
\hline Indiferente & 130 & 34,1 & $34,28(6,81)$ \\
\hline Buena & 137 & 36,0 & $36,31(7,02)$ \\
\hline Excelente & 2 & 0,5 & $26,50(2,12)$ \\
\hline TOTAL & 381 & 100.0 & $34,49(7,01)$ \\
\hline
\end{tabular}

El perfil del alumnado es predominantemente el de mujer (61,7\%), de 30 a 39 años (46,5\%), del área de Economía $(24,1 \%)$, que siente la docencia como vocacional $(81,6 \%)$, sin un interés concreto a corto plazo para interesarse por el Máster de Formación de Profesorado de Secundaria (55,9\%), sin experiencia docente $(34,6 \%)$, que considera aceptable la formación actual del Máster $(64,6 \%)$, por lo que no considera necesario ampliarla $(59,1 \%)$ y no cursaría estudios mayores para ello $(58,8 \%)$, y considera la imagen del docente, tanto la propia $(75,3 \%)$ como la de la sociedad (36\%), es predominantemente buena.

Respecto a la edad de los participantes, llama la atención como los participantes del área de Inglés $(X: 30,20 ; D T: 6,44)$ o los que consideran que la imagen social de los docentes es extrema, es decir, pésima (X: 31,00; DT: 6,40) o excelente (X: 26,50; DT: 2,12), son sensiblemente más jóvenes que el resto de opciones. Por otro lado, también destacan en sentido contrario los participantes del área de Tecnología $(X$ : $37,52 ; D T: 6,21)$, o Geografía e Historia $(X: 37,72 ; D T: 8,21)$ y los que tienen más de 5 años de experiencia docente $(X: 38,2 ; D T: 7,66)$.

En relación a los análisis inferenciales, la Tabla 2 muestra el análisis de significación de las distintas correlaciones entre variables nominales y ordinales mediante Chi-Cuadrado y $d$ de Somers sobre Tablas de Contingencia. Se debe tener en cuenta que para conseguir reflejar adecuadamente la orientación de los datos a través de la $d$ de Somers se ha atribuido valores de carácter ordinal a las variables nominales. Por ejemplo, para la variable sexo, mujeres 0 y hombres 1 , y en el caso de las variables dicotómicas con condiciones SÍ/NO, la condición Sí se refleja con el valor 1 y la condición $\mathrm{NO}$ con el valor 0. 
Tabla 2

Tabla de contingencia empleando la prueba de Chi-cuadrado (primer valor en cada celda) y D de Somers ( segundo valor en cada celda)

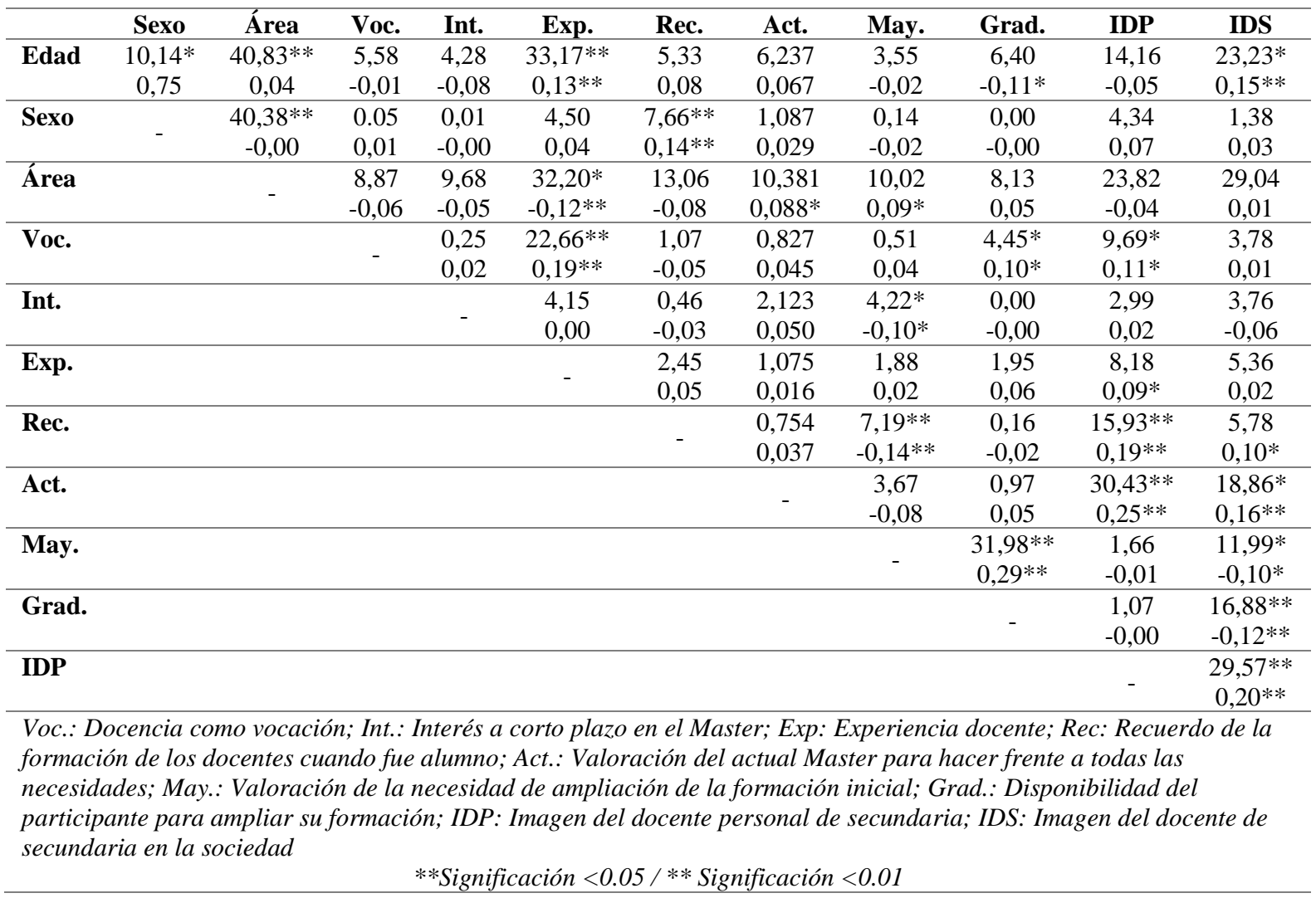

En la tabla 2 puede observarse cómo la distribución de las edades de los hombres en los distintos rangos de edades considerados al transformar la variable Edad a variable ordinal con cuatro niveles es significativamente diferente a la de las mujeres; puesto que la diferencia es significativa según ChiCuadrado, pero no hay una orientación significativa reflejada a través de la d de Somers. Esto no significa que los hombres sean significativamente mayores respecto a las mujeres, sino que sus agrupaciones por edades son distintas. Esto suele suceder cuando existen factores o condicionantes sociales distintos asociados al sexo del participante que afectan al estudio y no son incluidas dentro de su diseño inicial como variables intervinientes. Algo comprensible, puesto que es imposible incluir infinitos condicionantes sociales en el diseño del estudio para aislar el efecto del factor sexo.

Otro resultado destacable relacionado con la variable Sexo, es que los hombres tienen mucho mejor recuerdo de los profesores de secundaria cuando ellos fueron alumnos, lo que refleja fuertes diferencias de género en dicha asociación de variables. Respecto a la imagen de los docentes en la sociedad, los que creen que la sociedad tiene una imagen positiva del docente también suelen tenerla ellos mismos, tienen más edad, la formación recibida en el Master de Formación del Profesorado de Secundaria les parece suficiente y aceptable, por lo que no creen que haga falta ampliarlo, y no ampliarían sus estudios para ello.

Además de considerar que la imagen social de los docentes es positiva, los que tienen una imagen docente personal positiva sienten la docencia como vocacional, recuerdan positivamente a sus profesores de secundaria y les parece adecuada la formación actual. Por lo que respecta a la experiencia docente, se constata como edad y experiencia docente están fuertemente relacionadas; relación que puede considerarse esperable; y sienten la docencia como vocacional en sus vidas.

En relación a la posibilidad de ampliar la formación inicial de los docentes de secundaria, además de todo lo previamente expuesto, los que opinan que debe haber una formación mayor estarían dispuestos 
a ampliar sus estudios formativos, relación que puede considerarse esperable; no tienen un interés claro a corto plazo en la obtención del título del Master de Formación del Profesorado de Secundaria y tienen un recuerdo más negativo de los docentes de secundaria cuando ellos fueron alumnos.

En referencia a los que sienten la docencia como vocacional, aparte de las relaciones con otras variables ya comentadas, en mayor medida estarían dispuestos a ampliar su formación inicial para convertirse en docentes de secundaria. Por ejemplo, con la ampliación de la formación al formato de estudio de Grado Universitario.

Por último, se deja para el final la interpretación de la variable Área Académica, puesto que es la única variable nominal no transformable en ordinal, al ser ocho condiciones diferentes, por lo que la d de Somers no es capaz de mostrar información útil para su análisis. Será necesario, por lo tanto, acudir a la interpretación de cada una de las relaciones significativas según Chi-Cuadrado por separado a través de una comparativa general mediante porcentajes o mediante estadísticos descriptivos.

Según Chi-Cuadrado, existen diferencias significativas en edad, sexo y experiencia docente. Por lo que respecta a la edad, es observable en la Tabla 1 como los participantes del área de inglés (X: 30,20; DT: 6,44) destacan por ser los más jóvenes y, por otro lado, los participantes del área de Tecnología (X: 37,52; DT: 6,21) y de Geografía e Historia (X: 37,72; DT: 8,21) por ser considerablemente mayores.

En relación a la variable Sexo, la Figura 3 muestra los porcentajes de distribución de cada área según sexo, reflejando notables diferencias. Las mujeres muestran una distribución muestral considerablemente mayor a la media general $(61,7 \%)$ en Economía $(72,83 \%)$, Biología y Geología $(77,78 \%)$, Ingles $(80 \%)$ y Lengua y Literatura $(79,17 \%)$, y, por el contrario, los hombres muestran una distribución muestral considerablemente mayor a la media general $(38,3 \%)$ en Tecnología $(62,96 \%)$ y Matemáticas $(50,94 \%)$.

\section{Figura 3.}

Distribución de la muestra según área académica y sexo.

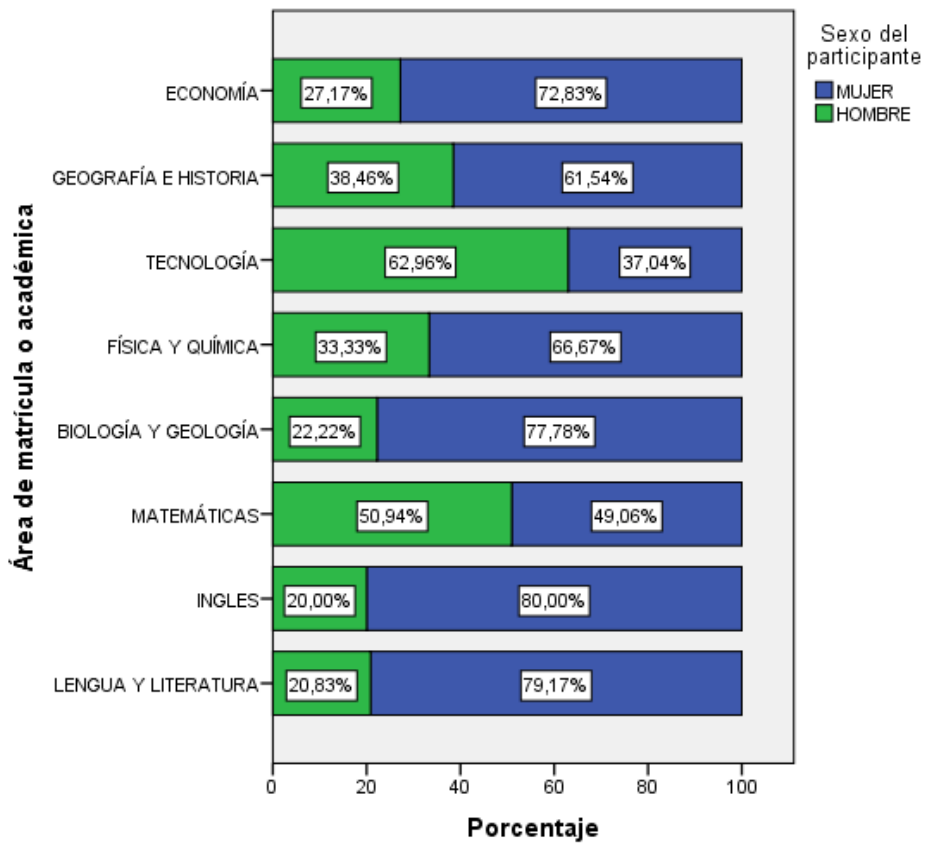

Finalmente, la Figura 4 muestra los porcentajes de distribución de cada área según la experiencia docente, reflejando notables diferencias respecto a la media general de la condición de Ninguna $(34,6 \%)$, que refleja cómo es muy poco frecuente entre los participantes de Inglés (10\%), Física y Química (18,52\%) y Lengua y Literatura (25\%) y muy habitual específicamente entre los de economía $(44,57 \%)$ y Geografía e historia $(43,59 \%)$. 


\section{Figura 4.}

Distribución de la muestra según área académica y experiencia docente.

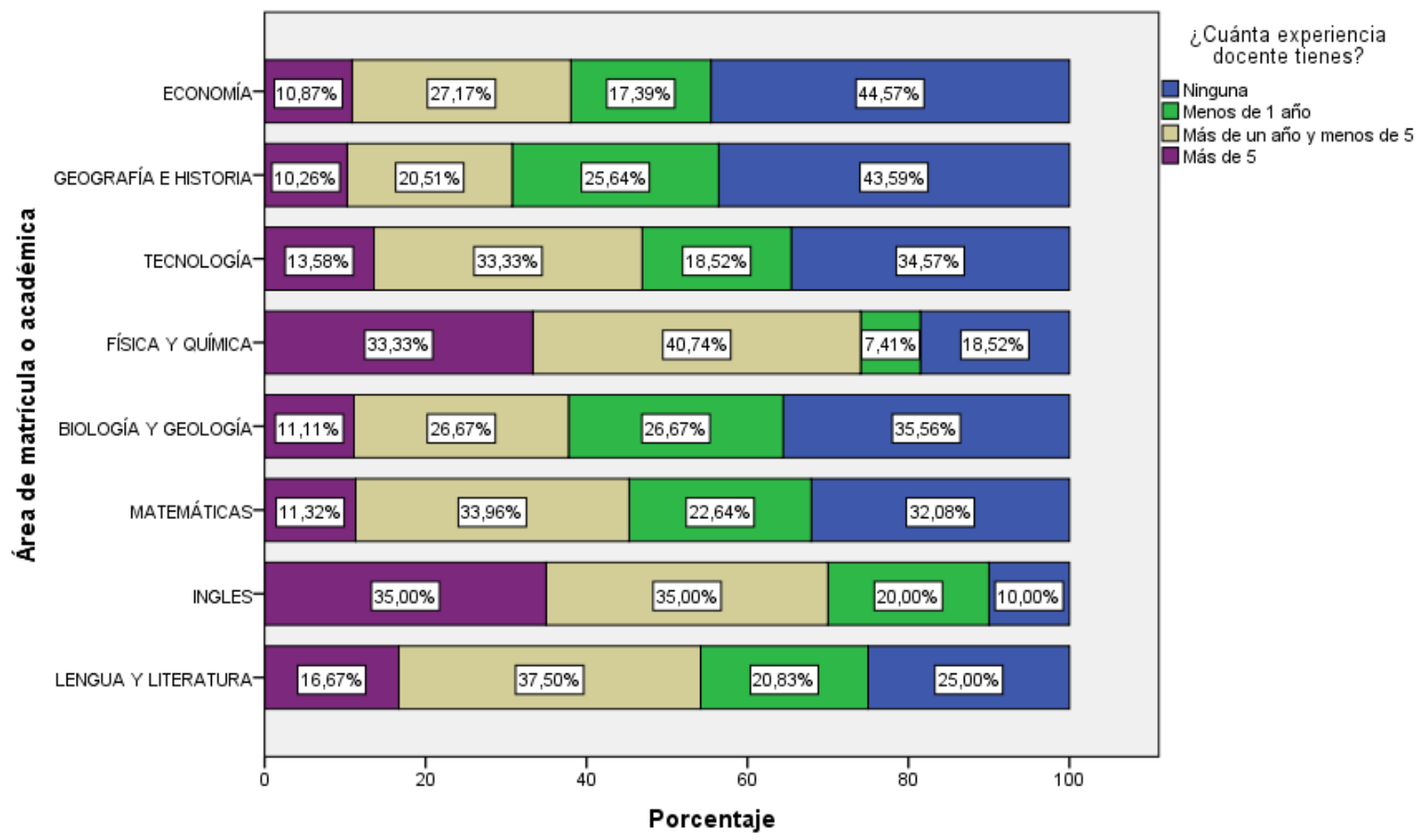

Conclusiones y Discusión

Los datos reflejan que el perfil social de los alumnos del Máster de Formación de Profesorado es mayoritariamente femenino, con una edad media en torno a los 35 años. Dato que concuerda con el estudio de Muñoz et al. (2019) donde constatan que el alumnado está formado mayoritariamente por mujeres (62\%). Una gran mayoría de los futuros docentes de secundaria consideran su interés en la docencia como vocacional y casi la mitad de ellos realiza el Master de Formación del Profesorado con intenciones laborales claras a corto plazo (oposiciones a empleo público u oferta laboral privada).

Llama la atención el contraste existente entre el gran porcentaje de alumnos del Máster de Formación de Profesorado que considera que su interés es vocacional $(81,6 \%)$ y una media de edad de inicio de la formación tan alta $(\mathrm{M}=34,49 ; \mathrm{DT}=7,01)$, junto a un porcentaje significativo de alumnos sin experiencia docente previa $(34,6 \%)$. Estos datos parecen indicar que el perfil de una parte significativa del alumnado del Máster de Formación del Profesorado es el de profesional de áreas no relacionadas directamente con la docencia con amplia experiencia laboral no docente, que trata de reciclarse desde otra área profesional a la que considera su vocación profesional real. Es importante señalar que, tal y como señala el Informe TALIS (OCDE, 2019), el 62\% de los docentes en España no escogió la enseñanza como primera opción para dedicarse profesionalmente (67\% en la OECD). Cabría preguntarse el porqué de que haya tantos docentes vocacionales que se alejan de las aulas durante sus primeros años de labor profesional, lo cual puede estar ligado a un posible bajo estatus social del docente en España (Zapico, et al., 2017).

Respecto al perfil formativo del alumnado del Máster de Formación de profesorado, se puede afirmar que las áreas más habituales son principalmente la Tecnología, predominantemente masculina, y la Economía, predominantemente femenina. El alumnado más joven es el del área de inglés y el mayor es el del área de Tecnología y Geografía e Historia. Por último, en relación a la experiencia docente, la mayoría del alumnado de Inglés o Física y Química tiene experiencia docente, mientras que en el área de Economía o Geografía e Historia la mayoría carece de experiencia docente. Que el perfil de alumnado de Economía sea el de personas más maduras y sin experiencia docente seguramente se deba a que el perfil laboral de dichas áreas suele llevarlos al ámbito empresarial y, por lo tanto, alejarles de las aulas inicialmente. El hecho de que el alumnado del área de Economía sean predominantemente mujeres puede 
indicar que esta área laboral no termina de satisfacer a largo plazo a las mujeres, por lo que se deciden a darle un cambio de rumbo a sus vidas y trasladar el perfil profesional hacia la docencia. Estos datos podrían estar describiendo un problema de 'Techo de cristal' (Adams y Funk, 2012; Glass y Cook, 2016) para las mujeres en el tejido empresarial, que les lanza a aventurarse a cambiar su área profesional hacia la docencia, pero también puede estar describiendo una insatisfacción con el área profesional entre las mujeres del área de Economía a largo plazo. Para distinguir entre las dos posibles causas de este resultado sería necesario abordar nuevas y más profundas investigaciones que la que aquí se describe.

En relación a la imagen social del docente, cabría destacar que uno de cada tres guarda mal recuerdo de la formación de los docentes de secundaria cuando fueron alumnos y aproximadamente dos de cada tres consideran que la sociedad no tiene buena opinión de los docentes en general. Resultados que coinciden con los obtenidos por Martínez y Villardón (2014) y Zamora y Cabrera (2015) en los que los docentes manifiestan que no tienen el prestigio social que merecen y que la profesión debería estar mejor valorada.

Los datos muestran que una inmensa mayoría $(97,6 \%)$ ven al menos adecuada la formación que reciben en el Master de Formación del Profesorado, aunque un 40\% percibe necesidad de ampliación y estarían dispuestos a cursar esa ampliación. Estos resultados guardan relación con la investigación realizada por Manso y Martín (2014) donde el alumnado encuestado indicó que se encuentra relativamente satisfecho con la formación recibida en el citado Máster, y con Martínez y Villardón (2015): "los estudiantes afirman que la formación recibida en el Máster ha modificado su concepción de la profesión en el sentido de que les ha hecho más conscientes de las funciones a desempeñar" (2015, p.465). Sin embargo, no guardan correspondencia con los datos, menos positivos, que se ponen de manifiesto en Sans de Castro (2013). Quizás, un punto medio se encuentra en el estudio de Serrano y Pontes (2015b) donde tras analizar esta temática se sitúan en una posición intermedia.

Los resultados muestran que existe una marcada diferenciación por sexo en relación a la percepción de la formación del docente desde la visión personal como alumno de secundaria en el pasado. Las mujeres muestran significativamente peor recuerdo de la formación de los docentes de secundaria, lo que podría estar indicando un margen de mejora formativo de los docentes de secundaria para afrontar los problemas más habituales asociados al rol femenino en la adolescencia (cambios corporales, presión social excesiva hacia la imagen, trastornos de la alimentación, etc.). Sería necesario una nueva investigación que evalúe la actualidad de dicho enunciado a través de la recogida de respuestas a esta misma cuestión entre los estudiantes de secundaria actuales. Si los resultados fueran similares, sería necesario considerar mejorar la formación de los docentes de secundaria hacia la comprensión, atención e intervención de los problemas sociales propios de las adolescentes para atender a esa destacable carencia.

Aparte de la relación directa y significativa con la imagen social del docente, la imagen del docente que tiene a nivel personal es significativamente más positiva si valora su interés en la docencia como vocacional, guarda buen recuerdo como estudiante de los docentes de secundaria y considera su formación actual en el Master de Formación del Profesorado como suficiente. Relaciones entre variables en cierto punto esperables, puesto que una mejor impresión de la docencia debe conllevar buenos recuerdos de sus docentes y de su propia formación.

La consideración de la necesidad de ampliar la formación de los docentes de secundaria correlaciona significativamente con un recuerdo negativo de los docentes de secundaria en la época de estudiante, con un interés en el Master de Formación del Profesorado no definido a corto plazo y con estar dispuesto a cursar mayores estudios para poder dedicarse profesionalmente a la docencia en secundaria. Estos resultados parecen ser coherentes con los intereses particulares de los participantes del estudio, puesto que, lógicamente, parece más probable que un alumno acepte una formación mayor si no tiene un empleo a corto plazo en objetivo, entre otros factores.

El interés vocacional en la docencia correlaciona significativamente con una mayor experiencia docente previa y con estar dispuesto a cursar mayores estudios para poder dedicarse profesionalmente a la docencia en secundaria. Sobre este dato, y sin pretender profundizar sobre el concepto de vocación, es interesante el estudio de Marchesi y Díaz (2007) en el que analizan las opiniones de los futuros docentes de Primaria y Secundaria, poniendo en valor que el 90,2\% de los encuestados afirma que para ser docente 
hace falta tener vocación. En este contexto, y puesto que en nuestro estudio se pone de manifiesto que hay un $40 \%$ de alumnos que estarían dispuestos a aumentar su formación (los cuales son predominantemente vocacionales y perciben con claridad la necesidad de formarse más para atender adecuadamente a los alumnos de secundaria), se plantea como propuesta de mejora la ampliación del Máster de Formación del Profesorado.

En base a dicha propuesta, podría esperarse que, unido a un proceso de selección del profesorado adecuado, se generen titulados posiblemente en mayor proporción vocacionales y terminarían teniendo una mejor formación que mejoraría la calidad de la educación a medio plazo, requisito fundamental, como se expuso en la introducción, para mejorar la calidad educativa. Esta propuesta se encuentra en sintonía con un dato importante del conocido Informe TALIS (OECD, 2019), en el que se señala que solo el $48 \%$ de docentes españoles afirma que recibió formación sobre el contenido de las materias, la pedagogía y la práctica en el aula, frente al 79\% de los países de la OECD.

Por último, como limitaciones principales de este estudio entendemos que estriban principalmente en que tal vez estos resultados podrían ser mejor explicados a través de estudios longitudinales, no transversales como éste, igualmente, aunque la muestra es relativamente amplia y el grado de generalización geográfica es significativo, ya que son alumnos de diferentes zonas de España, la muestra de estudiantes está circunscrita a una sola Universidad.

Contribución de cada Autor: Conceptualización, R.S..; metodología, R.S.; validación, R.S.; análisis, R.S.; escritura del manuscrito, R.S. y F.J.P.; escritura, revisión y edición, R.S. y F.J.P.; supervisión, R.S. y F.J.P.

Financiación: Este trabajo fue elaborado y financiado en el marco del proyecto «Profesionalización Docente: Discursos, políticas y prácticas. Nuevos enfoques y propuestas» (REF PID2020-112946GB-I00 / AEI / 0.13039/501100011033). Programa Estatal de Generación de Conocimiento y Fortalecimiento Científico y Tecnológico del Sistema de I+D+i, del Plan Estatal de Investigación Científica y Técnica y de Innovación 20172020. Ministerio de Ciencia e Innovación de España.

Conflicto de Intereses: Los autores declaran que no tienen conflicto de intereses.

\section{Referencias}

Adams, R. y Funk, P. (2012). Beyond the glass ceiling: Does gender matter? Management Science, 58(2), 219235. https://doi.org/10.1287/mnsc.1110.1452

Álvarez, V., Asensio, I., Clares, J., del Frago, R., García, B., García, N., García, M., Gil, J., González, D., Guardia, S., Ibarra, M., López, R., Rodríguez, A., Rodríguez, G., Rodríguez, J., Romero, S. y Salmerón P. (2009). Perfiles docentes para el Espacio Europeo de Educación Superior (EEES) en el ámbito universitario español. RELIEVE-Revista Electrónica de Investigación y Evaluación Educativa, 15(1), 1-18. https://doi.org/10.7203/relieve.15.1.4187

Ball, D. y Forzani, F. (2009). The Work of Teaching and the Challenge for Teacher Education. Journal of Teacher Education, 60 (5), 497-511. https://doi.org/10.1177/0022487109348479

Barber, M. y Mourshed, M. (2008). How the World's Best-Performing School Systems Come Out On Top. Londres: McKinsey Company, Social Sector Office. https://www.mckinsey.com/industries/public-and-socialsector/our-insights/how-the-worlds-best-performing-school-systems-come-out-on-top

Benarroch, A., Cepero S. y Perales F. (2013). Implementación del Máster de Profesorado de Secundaria: metodología y resultados de su evaluación. Revista Eureka sobre Enseñanza y Divulgación de las Ciencias, 10(extra FIPS), 594-615. https://revistas.uca.es/index.php/eureka/article/view/2811

Beyer, L. y Zeichner, K. (2018). Teacher education in cultural context: Beyond reproduction. En Thomas S. Popkewitz (Ed.), Critical Studies in Teacher Education. Its Folklore, Theory and Practice (pp. 298-334). London: Routledge. https://doi.org/10.4324/9780429450150-11

Biesta, G., Priestley, M. y Robinson, S. (2015). The role of beliefs in teacher agency. Teachers and Teaching, 21(6), 624-640. https://doi.org/10.1080/13540602.2015.1044325

Cochran, M. y Zeichner, K. (Eds.) (2009). Studying teacher education: The report of the AERA panel on research and teacher education. Routledge. https://www.aera.net/Publications/Books/Studying-TeacherEducation-The-Report-of-the 
Perfil y percepciones de los estudiantes del Máster universitario en formación del profesorado de educación secundaria en España

Darling-Hammond, L. (2017). Teacher education around the world: What can we learn from international practice? European Journal of Teacher Education, 40(3), https://doi.org/10.1080/02619768.2017.1315399

Fernández, J., López, M., Cruz, J., Bascuñán, J., Mengual, S., García, S., Lloret, C. y Grau, R. (eds.) (2016). La formación inicial del profesorado de educación secundaria. Tirant Humanidades. https://doi.org/10.5944/reec.29.2017.18075

Glass, C. y Cook, A. (2016). Leading at the top: Understanding women's challenges above the glass ceiling. The Leadership Quarterly, 27(1), 51-63. https://doi.org/10.1016/j.leaqua.2015.09.003

Hendriks, M., Luyten, H., Scheerens, J., Sleegers, P. y Steen, R. (2010). Teachers' professional development: Europe in international comparison. Luxembourg: Office for Official Publications of the European Union. $\quad$ https://research.utwente.nl/en/publications/teachers-professional-development-europe-ininternational-compari

Manso, J. y Martín E. (2014). Valoración del Máster de Formación del Profesorado de Educación Secundaria: estudios de casos en dos universidades. Revista de Educación, 364, 145-169.

Manso, J., Matarranz, M. y Valle, J. M. (2019). Estudio supranacional y comparado de la formación inicial del profesorado en la Unión Europea. Profesorado. Revista de Currículum y Formación de Profesorado, 23(3), 15-33. https://doi.org/10.30827/profesorado.v23i3.9697

Marcelo, C. (2010). La identidad docente: constantes y desafíos. Revista Interamericana de Investigación, Educación y Pedagogía, RIIEP, 3(1), 15-42. https://doi.org/10.15332/s1657-107x.2010.0001.01

Marchesi, A., y Díaz, T. (2007). Las emociones y los valores del profesorado. Cuadernos Fundación SM, 5, 1128.

Martínez, Z. y Villardón, L. (2014). La imagen profesional del profesorado en formación continua. Revista Fuentes, 15, 187-212

Martínez, Z. y Villardón, L. (2015). The image of a high school teacher in initial training. Profesorado. Revista de Currículum y Formación del Profesorado, $19(1), \quad 452-467$. https://recyt.fecyt.es/index.php/profesorado/article/view/41649

Muñoz, G., Rodríguez, P. y Luque, M. (2019). La formación inicial del profesorado de educación secundaria en España: perfil y motivaciones del futuro docente. Educación XX1, 22(1), 71-92. https://doi.org/10.5944/educxx 1.20007

OCDE (2019). TALIS 2018 Results (Volume I): Teachers and School Leaders as Lifelong Learners. París: TALIS, OECD Publishing. http://www.oecd.org/education/talis-2018-results-volume-i-1d0bc92a-en.htm

Perales, F., Cabo, J., Vílchez, J., Fernández, M., González, F. y Jiménez, P. (2014). La reforma de la formación inicial del profesorado de ciencias de secundaria: Propuesta de un diseño del currículo basado en competencias. Enseñanza de las Ciencias, 32(1), 9-28. https://doi.org/10.5565/rev/ensciencias.898

Pontes, A., Poyato, F. J. y Oliva, J. Mª (2015). Concepciones sobre el aprendizaje en estudiantes del Máster de profesorado de Educación Secundaria del área de ciencia y tecnología. Profesorado. Revista de $\begin{array}{lllll}\text { Currículum } y & \text { Formación } & \text { de } & \text { 225-243. }\end{array}$ https://recyt.fecyt.es/index.php/profesorado/article/view/40923

Rodríguez P., Muñoz, G. y Luque, M. (2019). Estudio exploratorio sobre el panorama actual de la formación inicial del profesorado de secundaria en España. IJERI: International Journal of Educational Research and Innovation, 11,169-184. https://www.upo.es/revistas/index.php/IJERI/article/view/2808

Romero, M. F. y Trigo E. (2018). Entre las creencias y la formación inicial de los estudiantes del Máster de Profesorado de Secundaria: una mirada hacia la enseñanza de la literatura en las aulas. Profesorado, Revista de Currículum y Formación del Profesorado, 22(1), 73-96. https://recyt.fecyt.es/index.php/profesorado/article/view/63622

Sánchez-Cabrero, R., Arigita-García, A., Barrientos-Fernández, A., León-Mejía, A. C. (2019). Online Explorative Study on the Learning Uses of Virtual Reality Among Early Adopters. Journal of Visualized Experiments, JOVE (153), e60188. https://dx.doi.org/10.3791/60188

Sans de Castro, R. (2013). Experiencia y valoración del máster de Secundaria. IBER: Didáctica de las Ciencias Sociales, Geografía e Historia, 73, 65-72.

Serrano, R. y Pontes, A. (2015a). Expectativas ante la formación inicial entre el alumnado del Máster de Profesorado de Enseñanza Secundaria. Revista de Investigación Educativa, 33(2), 489-505. https://doi.org/10.6018/rie.33.2.203471 
Serrano, R. y Pontes, A. (2015b). Nivel de desarrollo de las competencias y objetivos generales del Máster Formación del Profesorado de Enseñanza Secundaria. Perfiles educativos, 37(150), 39-55. https://doi.org/10.22201/iisue.24486167e.2015.150.53161

Sola, J.M., Marín, J.A., Alonso, S. y Gómez, G. (2020). Análisis de percepciones del estudiantado del Máster de Secundaria respecto a las competencias profesionales del docente. Revista Electrónica Interuniversitaria de Formación del Profesorado, 23(2), 81-93. https://doi.org/10.6018/reifop.418601

Zamora, B. y Cabrera, L. (2015). La sociedad y el profesorado. Imágenes y opiniones sociales sobre el profesorado. RASE: Revista de la Asociación de Sociología de la Educación, 8 (1), 86 - 107. https://ojs.uv.es/index.php/RASE/article/view/8763

Zapico, M. H., Martínez, E. y Montero, M. L. (2017). Demandas sociales y formación inicial del profesorado: ¿Un callejón sin salida? Profesorado, Revista de Currículum y Formación del Profesorado, 21(1), 80-102. https://recyt.fecyt.es/index.php/profesorado/article/view/58051 